\title{
Access Delay Analysis of IEEE 802.11 DCF in the Presence of Hidden Stations
}

\author{
Fu-Yi Hung and Ivan Marsic \\ CAIP Center, ECE Department, Rutgers University \\ Piscataway, NJ 08854-8088 \\ \{fuyihung,marsic\}@caip.rutgers.edu
}

\begin{abstract}
In this paper, we present an analytical model to evaluate the hidden station effect on the access delay of the IEEE 802.11 Distributed Coordination Function (DCF) in both non-saturation and saturation condition. DCF is a random channel-access scheme based on Carrier Sense Multiple Access with Collision Avoidance (CSMA/CA) method and the exponential backoff procedure to reduce packet collisions. However, hidden stations still cause many collisions under CSMA/CA method because stations cannot sense each other's transmission and often send packets concurrently, resulting in significant performance degradation. Prior research has built accurate access delay model for 802.11 DCF. However, the hidden station effect on the performance has not been adequately studied. Our model generalizes the existing work on access delay modeling of 802.11 DCF for both non-saturation and saturation conditions, under the hidden-station effect. The performance of our model is evaluated by comparison with ns- 2 simulations and they are found to agree.
\end{abstract}

\section{INTRODUCTION}

IEEE 802.11 [1] is the most popular standard used in Wireless Local Area Networks (WLANs). The IEEE 802.11 Medium Access Control (MAC) defines two access methods: the Distributed Coordination Function (DCF) and the Point Coordination Function (PCF). The polling-based PCF uses a virtual carrier-sense mechanism aided by an access priority mechanism to control the channel access. On the other hand, the contention-based DCF uses a random access scheme where each station has the right to initiate its transmission without infrastructure support. So, this scheme is useable not only in infrastructure network configurations but also in distributed and self-organized wireless networks. The support for various wireless networks has made the DCF very popular. The basic access mechanism is a two-way handshaking method where the sender transmits a data frame and the receiver replies with an acknowledgement (ACK) frame to confirm a successful transmission. However, it unavoidably suffers from the hidden station problem [2-4] because of multiple simultaneous transmissions on the same channel without any coordinator. In order to prevent the interference from hidden stations, DCF introduces Request-To-Send/ Clear-To-Send (RTS/CTS) access mechanism. RTS/CTS, which is a four-way handshaking method, reserves the medium before transmitting a data frame by transmitting a RTS frame and replying a CTS frame.

The modeling of IEEE 802.11 has attracted a number of studies. Ref. [5] was the first to derive a model that incorporates the exponential backoff process inherent to 802.11 as a two dimensional Markov chain. Ref. [6] follows the same Markov chain model and considers frame retry limits to avoid overestimating the throughput of 802.11 as in [5]. Ref. [7] extends this model to evaluate the saturated throughput under the hidden station effect. Refs. [8, 9] modified this model to represent the non-saturation and saturation conditions. On the other hand, an average service time and jitter analysis in the saturated condition is derived in [10] based on this Markov chain model. Ref. [11] considers the data length and the number of backoff slot as random variables. It proposes a probability generating function (PGF) for the access delay and inverts this PGF to obtain an approximate distribution of the access delay. Refs. [12, 13] extend this PGF work by considering more parameters as random variables to derive their PGFs and distributions for the access delay.

Prior research has attempted to build an accurate access delay model for 802.11 DCF. However, the hidden station effect on the backoff scheme has not been given adequate attention. As will be shown below, this effect significantly increases the access delay, especially for the basic access method in the saturated condition. In this paper, we study the access delay of 802.11 DCF under non-saturation and saturation conditions in the presence of hidden stations for both basic and RTS/CTS access methods based on the Markov chain model and the PGF analysis. The rest of this paper is organized as follows. In section II, we introduce the concept of vulnerable period to study the effectiveness of both basic and RTS/CTS access methods in the presence of hidden stations. In section III, we use a two-dimensional Markov chain model to calculate the packet transmission probability in the vulnerable period and the access delay in both non-saturation and saturation condition. In section IV, we validate the analytical model by comparing the numerical results with ns- 2 simulation [14]. Finally, conclusions are presented in section V.

\section{The Hidden StATION EFFECT ON 802.11 DCF}

The DCF is the fundamental access method of the IEEE 802.11. It is based on the CSMA/CA and a backoff procedure to reduce the collision probability between multiple stations accessing the channel. The CSMA/CA mechanism defines two channel states: idle and busy. If a station senses no transmission on the channel, it considers the channel state as idle; otherwise it considers the channel state as busy. When a station tries to access the channel, it enters the backoff procedure that randomly chooses a backoff time in a range $(0$, $C W_{0}$ ) with a uniform probability. The $C W_{0}$ is known as the minimum contention window size. During the backoff procedure, if the station senses channel as idle its timer decrements one backoff slot. If the channel is sensed as busy, 


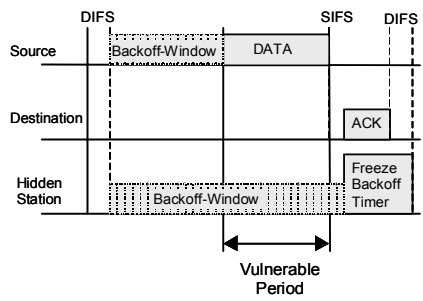

Fig. 1.The vulnerable period for the hidden stations: Basic access method

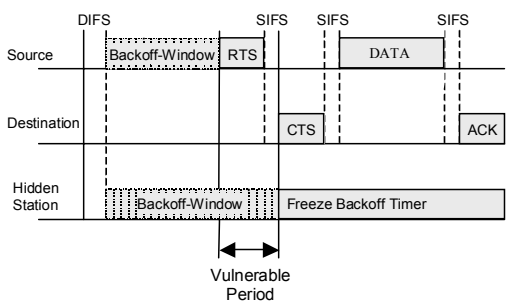

Fig. 2.The vulnerable period for the hidden stations: RTS/CTS access method

the timer is frozen. After the channel becomes idle again, the timer resumes from the frozen slot and counts down the remaining backoff slots. After the timer finishes the countdown, the station accesses the channel again. If the transmission fails, the station repeats the backoff procedure and doubles the contention window size. After every failed transmission, the exponential backoff mechanism doubles the contention window size up to a predefined maximum range. However, if some stations are hidden to each other so they cannot sense each other's transmission, they may mistakenly determine the channel as idle and transmit concurrently. The period from the end of the previous transmission until an ongoing transmission is detected is called the vulnerable period.

\section{A. Hidden Station Effect on the Basic Access Method}

The basic access method is shown in Fig. 1. Any station that can sense the transmission from the source, called covered station, will determine the channel as busy and defer its own transmissions for the duration of the Network Allocation Vector (NAV). The only possible packet collision between the source and a covered station happens if they finish their backoff countdown simultaneously. The vulnerable period for covered stations is one backoff slot long. However, the hidden stations do not sense the transmission from the source until they receive an ACK, so they sense the channel as idle until sensing the ACK. If any one of these hidden stations completes its backoff procedure before sensing the ACK, it will send another data frame to the destination, which will collide with the data frame from the existing source. The vulnerable period for hidden stations equals the length of a data frame.

\section{B. Hidden Station Effect on the RTS/CTS Access Method}

The RTS/CTS access method is shown in Fig. 2. As in the basic access method, the vulnerable period for the covered stations is also one backoff slot long. The hidden stations will set their NAV after receiving the CTS frame from the destination, so the vulnerable period for the hidden stations

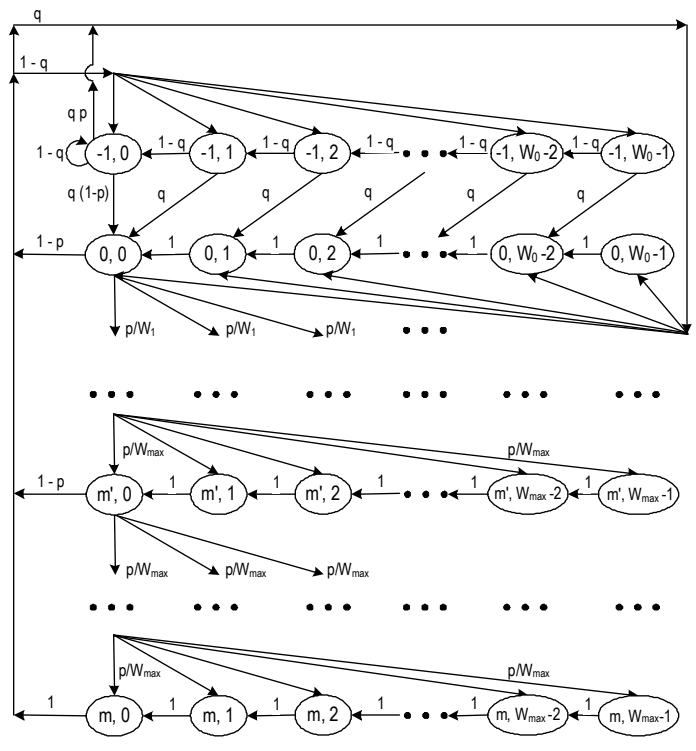

Fig. 3. Markov chain model for backoff procedure

equals the length of the RTS frame plus a SIFS period. Unlike the basic access method, the vulnerable period for hidden stations in RTS/CTS access method is a fixed length period and is not related to the length of the data frame from the source.

\section{The ACCess Delay Model}

The key contribution of this paper is the combined analytical evaluation of the access delay for both non-saturated and saturated conditions in the presence of hidden stations.

\section{A. Markov Chain Model of Station Transmissions}

In the analysis, we assume the following conditions: (a) ideal channel condition, i.e., no capture effect; (b) constant and independent collision probability of a packet transmitted by each station, regardless of the number of collisions already suffered; and (c) fixed number of contending stations.

Let $n$ denote the number of contending stations. Let $b(t)$ be the stochastic process representing the backoff timer for a given slot. As in [5], the key approximation in this model is that the probability $p$ of a transmitted packet colliding with another packet is independent of the station's backoff stage $s(t)$. So, the two-dimensional process $\{s(t), b(t)\}$ can be modeled as a discrete-time Markov chain, shown in Fig. 3, where $m$ is the maximum backoff stage and $m^{\prime}$ is the backoff stage at which the contention window size reaches the maximum value, $C W_{\max }$, and remains at $C W_{\max }$ after this stage. $W_{0}=\left(C W_{\min }+1\right)$ and $W_{m}=\left(C W_{\max }+1\right)$. The $C W_{\min }$ is the minimum contention window size. We set $m=m^{\prime}=5$ in this paper.

Based on the 802.11 standard [1], the contention window, also called backoff window, increases exponentially from $C W_{\min }$ to $C W_{\max }$. It can be represented as

$$
W_{i}=\left\{\begin{array}{cc}
W_{0} & i=-1 \\
2^{i} W_{0} & 0 \leq i \leq m^{\prime} \\
2^{m^{\prime}} W_{0} & i>m^{\prime}
\end{array}\right.
$$

The backoff states $(-1, k)$ for $k \in\left(0, W_{0}-1\right)$ in Fig. 3 represent the post-backoff stage. After a successful 


$$
\begin{aligned}
& b_{0,0}=\left\{\frac{1}{2} \cdot\left(\frac{1-p^{m+1}}{1-p}\right)+\frac{W_{0}}{2} \cdot\left(\frac{1-(2 p)^{m+1}}{1-(2 p)}\right)+\left(p \cdot \frac{W_{0}-1}{2}+\frac{1}{q W_{0}}\right) \cdot\left(\frac{(1-q)-(1-q)^{W_{0}+1}}{q}\right)\right\}^{-1} \\
& \tau_{2}=\sum_{i=0}^{m} \sum_{k=0}^{V} b_{i, k}= \\
& =\left\{1+(V+1)\left(\frac{p-p^{m+1}}{1-p}\right)-\frac{V(V+1)}{2 W_{0}} \cdot\left(\frac{\left(\frac{p}{2}\right)-\left(\frac{p}{2}\right)^{m+1}}{1-\left(\frac{p}{2}\right)}\right)-\frac{V(1-q)}{q W_{0}}+\frac{1}{W_{0}}\left(\frac{(1-q)^{W_{0}-V+1}-(1-q)^{W_{0}+1}}{q^{2}}\right)+\frac{V\left(2 W_{0}-V-1\right)}{2 W_{0}}\left(1+p\left(\frac{(1-q)-(1-q)^{W_{0}+1}}{q}\right)\right)\right\} \cdot b_{0,0} \quad, V \leq W_{0}
\end{aligned}
$$

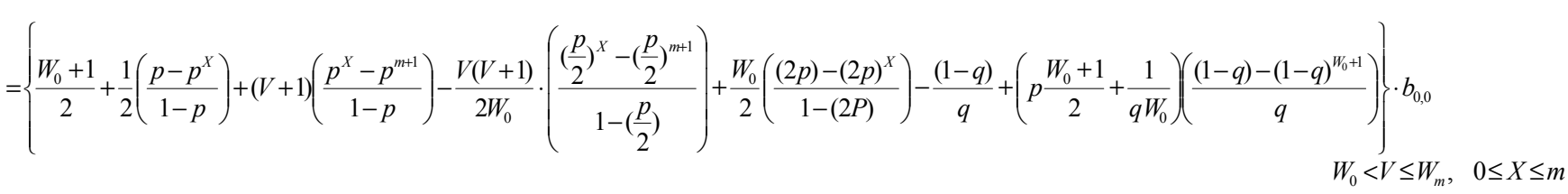

$=1, V>W_{m}$

transmission, the station resets its $\mathrm{CW}$ value to $\mathrm{W}_{0}$ and performs a random backoff procedure even if there are no pending packets in the queue. This post backoff ensures that there is at least one backoff interval between two consecutive transmissions of a station. We assume that each station can buffer one packet and $\mathrm{q}$ represents the probability that at least one packet waits for transmission during a slot time. If $q=1$, then the station is in the saturation condition and does not go to post-backoff stage. In this Markov chain, the transition probabilities are

$$
\begin{array}{ll}
P(-1,0 \mid-1,0)=1-q & \\
P(0,0 \mid-1,0)=q(1-p)+\frac{q p}{W_{0}} & \\
P(0, k \mid-1, k)=\frac{q p}{W_{0}} & 1 \leq k \leq W_{0}-1 \\
P(-1, k-1 \mid-1, k)=1-q & 1 \leq k \leq W_{0}-1 \\
P(0, k-1 \mid-1, k)=q & 1 \leq k \leq W_{0}-1 \\
P(-1, k \mid i, 0)=\frac{(1-q)(1-p)}{W_{0}} & 0 \leq k \leq W_{0}-1, \quad 0 \leq i \leq m-1 \\
P(-1, k \mid m, 0)=\frac{1-q}{W_{0}} & 0 \leq k \leq W_{0}-1 \\
P(0, k \mid i, 0)=\frac{q(1-p)}{W_{0}} & 0 \leq k \leq W_{0}-1, \quad 0 \leq i \leq m-1 \\
P(0, k \mid m, 0)=\frac{q}{W_{0}} & 0 \leq k \leq W_{0}-1 \\
P(i+1, k \mid i, 0)=\frac{p}{W_{i+1}} & 0 \leq k \leq W_{i+1}-1, \quad 0 \leq i \leq m-1 \\
P(i, k-1 \mid i, k)=1 & 1 \leq k \leq W_{i}-1, \quad 0 \leq i \leq m
\end{array}
$$

Let $b_{i, k}=\lim _{t \rightarrow \infty} P\{s(t)=i, b(t)=k\}, i \in(0, m), k \in\left(0, W_{i}-1\right)$ be the stationary distribution of the Markov chain. By using the normalization condition for a stationary distribution, we have

$$
1=\sum_{k=0}^{W_{0}-1} b_{-1, k}+\sum_{i=0}^{m} \sum_{k=0}^{W_{i}-1} b_{i, k}
$$

Based on the chain regularities, we can obtain $b_{0,0}$ in (4). The stationary probability $\tau 1$ (that a station transmits a packet in a randomly chosen time slot), can be represented as:

$$
\tau_{1}=\sum_{i=0}^{m} b_{i, 0}=\frac{1-p^{m+1}}{1-p} b_{0,0}
$$

The stationary probability $\tau_{2}$ (that a station transmits a packet in a vulnerable period), can be represented as Eq. (6) where $V$ is the vulnerable period length in the units of backoff slots. $X$ is the minimum backoff stage at which the backoff window size is greater than $V$. For example, if $W_{1}<V \leq W_{2}$, then use $X=2$ in Eq. (6). As already noted, $\tau_{1}$ is a special case of $\tau_{2}$ because $\tau_{1}$ can be considered as the vulnerable period with the duration of one slot time. We can verify this using $V=0$ and $X=0$ in the first case of Eq. (6).

In the stationary state, the collision probability $p$ is the probability that at least one covered station transmits in the same backoff slot as the source, or at least one hidden station transmits in the vulnerable period. Thus $p$ can be expressed as:

$$
p=1-\left(1-\tau_{1}\right)^{n_{C}-1}\left(1-\tau_{2}\right)^{n_{H}}
$$

where $n_{C}$ is number of the covered stations that includes the transmitting station itself, and $n_{H}$ is the number of the hidden stations. The total number of contending stations, $n$, equals $n=$ $n_{C}+n_{H}$. We solve the nonlinear Eqs. (4)-(7) by numerical method to obtain $\tau_{1}$ and $\tau_{2}$.

\section{B. The Access Delay Analysis}

Let $P_{t r}$ be the probability that there is at least one transmission in the considered slot time.

$$
P_{t r}=1-\left(1-\tau_{1}\right)^{n}
$$

The probability of a successful transmission in the presence of hidden stations, $P_{s}$, is the probability that exactly one of $n$ backlogged stations transmits and none of its covered station 
transmits in the same time slot and none of the hidden stations transmits in the vulnerable period

$$
P_{S}=\frac{n \tau_{1}\left(1-\tau_{1}\right)^{n_{C}-1}\left(1-\tau_{2}\right)^{n_{H}}}{P_{t r}}
$$

The length of a slot time $E S$ can be represented as

$$
E S=\left(1-P_{t r}\right) \sigma+P_{S} P_{t r} T_{S}+\left(1-P_{S}\right) P_{t r} T_{C}
$$

where $\sigma$ is the duration of an empty backoff slot. The $T_{S}$ and $T_{C}$ are the period of time that the channel is sensed busy because of a successful transmission or a collision, respectively. They are different in the basic and RTS/CTS access methods:

$$
\begin{aligned}
& T_{S}^{\text {bas }}=D A T A+\delta+S I F S+A C K+\delta+D I F S \\
& T_{C}^{\text {bas }}=D A T A+\delta+A C K{ }_{-} \text {Timeout }
\end{aligned}
$$

where $D A T A$ is the data frame length and $\delta$ is the propagation delay. The $A C K \_$Timeout $=S I F S+A C K+D I F S$. For RTS $/ C T S$ access method, $\bar{T}_{S}$ and $T_{C}$ can be expressed as:

$$
\begin{aligned}
T_{S}^{r t s}= & R T S+\delta+S I F S+C T S+\delta+S I F S \\
& +D A T A+\delta+S I F S+A C K+\delta+D I F S \\
T_{C}^{r t s}= & R T S+\delta+C T S \quad \text { Timeout }
\end{aligned}
$$

where CTS_Timeout $=S I F S+C T S+(2 \times \sigma)$. Let $U_{i}$ be a random variable with a discrete uniform distribution

$$
\operatorname{Pr}\left\{U_{i}=k\right\}=\frac{1}{W_{i}} \quad k=0,1, \ldots\left(W_{i}-1\right) \quad i=0,1, \ldots m
$$

The accumulated backoff time $B_{i}$ before a station transmits a packet successfully from the $i$ th backoff stage or discards it because of exceeding the retry limit $m$ can be represented as

$$
B_{i}=\left\{\begin{array}{lc}
E S \cdot \sum_{r=0}^{i} U_{r}+i \cdot T_{C} & i=0,1, \ldots m \\
E S \cdot \sum_{r=0}^{m} U_{r}+i \cdot T_{C} & i=m+1
\end{array}\right.
$$

The station discards the packet when the stage $m+1$ is reached. Hence, the probability of a station transmitting a packet successfully at $i$ th stage or discarding it at $m+1$ stage is $p_{i}$

$$
p_{i}=\left\{\begin{array}{cc}
(1-p) p^{i} & i=0,1, \ldots m \\
p^{i} & i=m+1
\end{array}\right.
$$

So the access delay $D$ including all backoff stages is

$$
D=\sum_{i=0}^{m+1} p_{i} \cdot B_{i}+T_{S}
$$

In order to obtain the distribution of the access delay, we derive the Probability Generating Function (PGF) of the access delay $D(Z)$ by $z$-transform [11-13]

$$
D(Z)=\sum_{i=0}^{m+1} p_{i} \cdot B_{i}(Z)+Z^{T_{S}}
$$

where the $B_{i}(Z)$ can be represented as

$$
B_{i}(Z)=\left\{\begin{array}{lc}
E S(Z) \cdot \sum_{r=0}^{i} U_{r}(Z)+i \cdot Z^{T_{C}} & i=0,1, \ldots m \\
E S(Z) \cdot \sum_{r=0}^{m} U_{r}(Z)+i \cdot Z^{T_{C}} & i=m+1
\end{array}\right.
$$

and $E S(Z)$ can be represented as

$$
E S(Z)=\left(1-P_{t r}\right) \cdot Z^{\sigma}+P_{S} P_{t r} \cdot Z^{T_{S}}+\left(1-P_{S}\right) P_{t r} \cdot Z^{T_{C}}
$$

We invert the PGF of the access delay numerically to obtain its distribution. The approximate distribution of the access delay is validated in next section.

\section{A. Simulation Setup}

\section{Performance Evaluation}

All the parameters used in the analytical model and the ns-2 simulation are summarized in Table I.

Table I. System parameters

\begin{tabular}{|l|l|}
\hline Transmission Rate & $1 \mathrm{Mbps}$ \\
\hline Packet Payload & 250 Bytes \\
\hline PHY header & 192 bits \\
\hline RTS & 160 bits + PHY header \\
\hline CTS & 112 bits + PHY header \\
\hline ACK & 112 bits + PHY header \\
\hline DIFS & $50 \mu \mathrm{s}$ \\
\hline SIFS & $10 \mu \mathrm{s}$ \\
\hline Slot Time $(\sigma)$ & $20 \mu \mathrm{s}$ \\
\hline Propagation Delay $(\delta)$ & $1 \mu \mathrm{s}$ \\
\hline$C W_{\min }$ & 31 \\
\hline$C W_{\max }$ & 1023 \\
\hline
\end{tabular}

We use a ring topology that is composed of one access point located in the center of a ring and 16 stations uniformly distributed along the ring. The capture effect can be ignored in our topology because of equal distance from the access point to all stations. The transmission range and carrier sensing ranges are set at 597 meters (m). In this study, we vary the ring diameter, defined as $d$, to obtain different number of hidden stations in the 16-station network: (a) $d=540 \mathrm{~m}$ - each station can sense all the packets from the other 15 stations, so there is no hidden stations and there are 16 covered stations; (b) $d=$ $600 \mathrm{~m}-1$ hidden station and 15 covered ones; (c) $d=630$ $\mathrm{m}-3$ hidden stations and 13 covered stations; (d) $d=680$ $\mathrm{m}-5$ hidden stations and 11 covered ones.

\section{B. Model Validation and Access Delay Analysis}

First, we validate the average access delay of our analytical model with the ns- 2 results for different offered load in both basic and RTS/CTS access methods, as shown in Figs. 4 and 5. Based on the result, the access delay can be divided into three conditions: non-saturation, transition and saturation. In the non-saturation condition, as the offered load increases, the access delay increases linearly in both access methods. Access delay values are almost the same regardless of the number of hidden stations in the network. The hidden station effect is insignificant in this condition. In the transition condition, the access delay jumps from its non-saturated value to the saturated one. As the number of hidden station increases, there is a higher overshoot in the access delay curve. The analytical access delay cannot capture the simulated result because we solve nonlinear Eqs. (4)-(7) by numerical method to get an approximate analytical result. In the saturation condition, the access delay remains constant. Comparing this value in both the basic and RTS/CTS access methods, we can see that the basic access method is much more sensitive to the hidden 


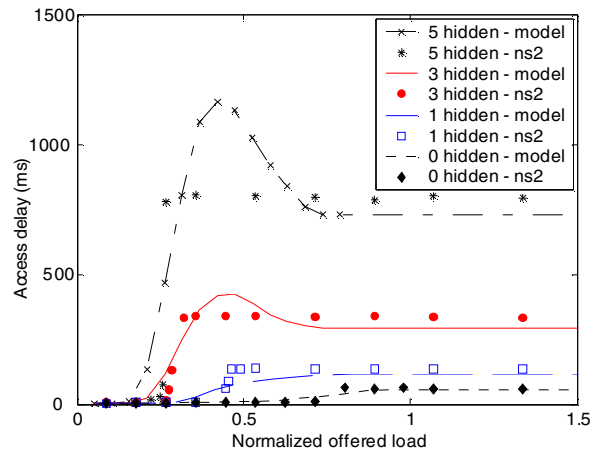

Fig. 4 Access delay versus normalized offered load: Basic access method

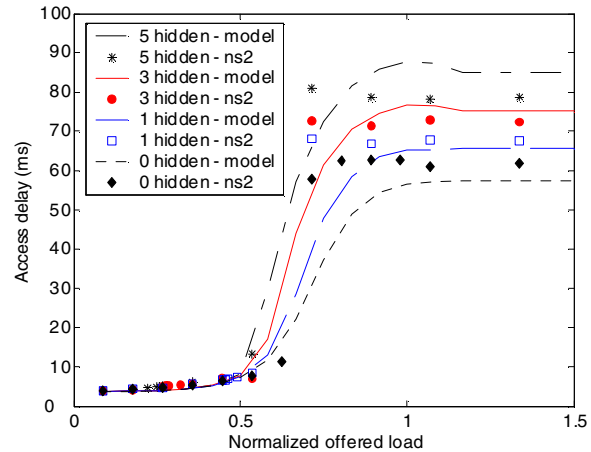

Fig. 5 Access delay versus normalized offered load: RTS/CTS access method

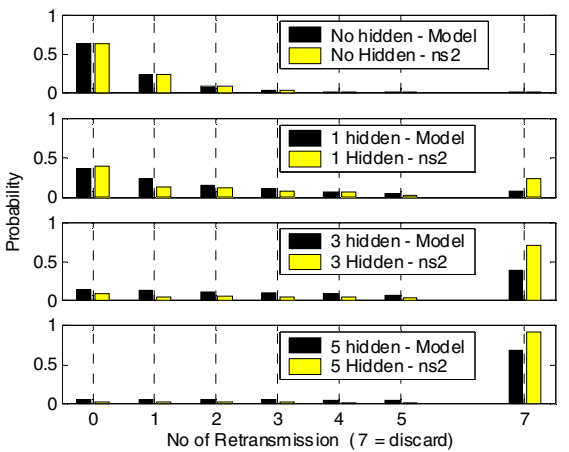

Fig. 6 PMF of number of retry Basic access method

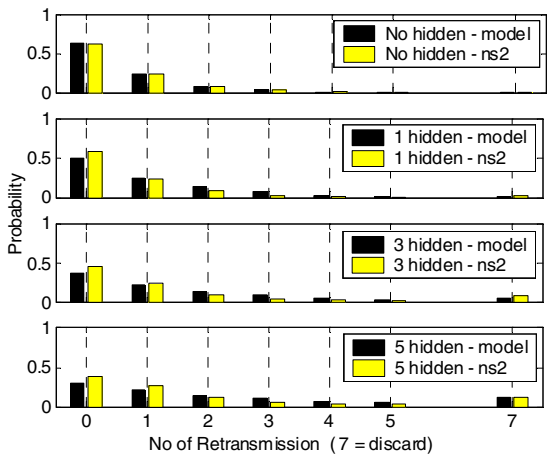

Fig. 7 PMF of number of retry RTS/CTS access method

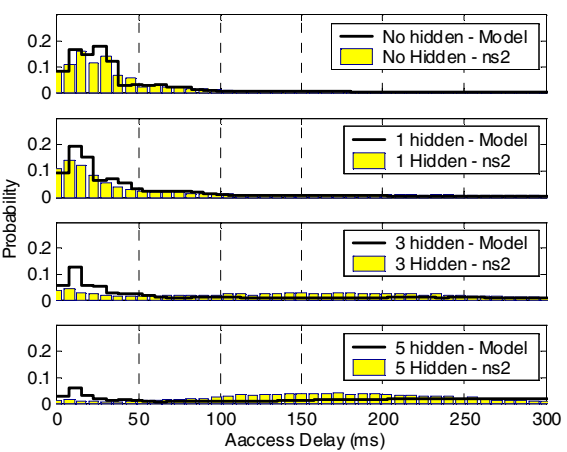

Fig. 8 PMF of the access delay Basic access method
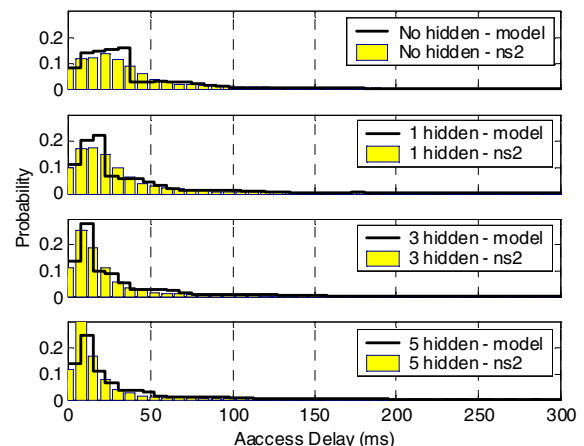

Fig. 9 PMF of the access delay RTS/CTS access method station effect. As the number of hidden station grows, the access delays in 1, 3 and 5 hidden station cases are about 2.3, 5.5 and 13 times of that in the case of no hidden stations, respectively. Since the previous 802.11 DCF delay models do not account for hidden stations, their delays would correspond to the bottom curves in Figs. 4 and 5 (no hidden stations scenario). Obviously, this is inadequate.

We also study the Probability Mass Function (PMF) of the number of retries before a successful transmission (Fig. 6 and 7) and access delay (Figs. 8 and 9) in the saturation condition. In the basic access method, as the number of hidden station grows, fewer packets can be transmitted successfully in the first attempt and more packets will be discarded because of exceeding the retry limit (Fig. 6). The distribution of the access delay (Fig.8) also shows this tendency. The PMF is getting flat and widespread as the number of hidden stations increases. On the other hand, the RTS/CTS access method is more robust to the hidden-station effect. The PMF charts for different network scenarios are similar (Figs. 7 and 9).

\section{CONCLUSION}

In this paper, we derived an analytical model for the access delay of the basic and RTS/CTS access methods in IEEE 802.11 DCF under both the non-saturation and saturation condition in the presence of hidden stations. The proposed model is in good agreement with ns-2 simulations and, thus, can be used to estimate accurately the access delay. The previous work can be considered as a special case of our model, with zero hidden stations.

\section{REFERENCES}

[1] IEEE Standard for Wireless LAN Medium Access Control (MAC) and Physical Layer (PHY) specifications, IEEE 802.11b, 1999.

[2] A. S. Tanenbaum, Computer Networks. 3rd Edition, Prentice Hall, Upper Saddle River, NJ, 1996.

[3] L. Kleinrock and F. Tobagi, "Packet switching in radio channels, Part II-The hidden terminal problem in carrier sense multiple access and the busy tone solution," IEEE Trans. Comm., vol. COM-23, no. 12, pp. 1417-1433, Dec. 1975.

[4] H. S. Chhaya and S. Gupta, "Performance modeling of asynchronous data transfer methods of IEEE 802.11 MAC protocol," Wireless Networks, vol. 3, pp. 217-234, 1997.

[5] G. Bianchi, "Performance analysis of the IEEE 802.11 distributed coordination function," IEEE J. on Selected Areas in Comm., vol. 18, no. 3, pp. 535-547, Mar. 2000.

[6] H. Wu, Y. Peng, K. Long, S. Cheng, and J. Ma, "Performance of reliable transport protocol over IEEE 802.11 wireless LAN: Analysis and enhancement," Proc. IEEE INFOCOM, vol. 2, pp. 599-607, 2002.

[7] F. Hung, S Pai and I. Marsic, "Performance Modeling and Analysis of the IEEE 802.11 Distribution Coordination Function in Presence of Hidden Stations," IEEE/AFCEA MILCOM, Oct. 2006.

[8] K. Duffy, D. Malone and D. Leith, "Modeling the 802.11 Distributed Coordination Function in Non-saturated Conditions," IEEE Comm. Letters. Vol. 9, No. 8, 715-717, Aug. 2005.

[9] P.E. Engelstad and O.N. Østerbø, "Non-Saturation and Saturation Analysis of IEEE 802.11e EDCA with Starvation Prediction," Proc. ACM MSWiM 2005, Oct. 2005.

[10] M. Carvalho and J.J. Garcia-Luna-Aceves, "Delay Analysis of IEEE 802.11 in Single-Hop Networks," Proc. IEEE ICNP 03, Nov. 2003.

[11] H. Zhai, Y. Kwon, and Y. Fang, "Performance analysis of IEEE 802.11 MAC protocols in wireless LANs," in Proc. Wireless Commun. And Mobile Computing 2004, pp. 917-931

[12] O. Tickoo and B. Sikdar, "Queueing analysis and delay mitigation in IEEE 802.11 random access MAC based wireless networks," in Proc. IEEE INFOCOM 2004, pp. 1404-1413

[13] H. Vu and T. Sakurai, "Accurate delay distribution for IEEE 802.11 DCF," in IEEE Communications Letters, Vol. 10, NO. 4, Apr. 2006.

[14] The ns Manual, http://www.isi.edu/nsnam/ns/ns-documentation.html 